\title{
Daily variation at Concordia station (Antarctica) and its dependence on IMF conditions
}

\author{
L. Cafarella ${ }^{1}$, D. Di Mauro ${ }^{1}$, S. Lepidi ${ }^{1}$, A. Meloni ${ }^{1}$, M. Pietrolungo ${ }^{1}$, L. Santarelli ${ }^{1}$, and J. J. Schott ${ }^{2}$ \\ ${ }^{1}$ Istituto Nazionale di Geofisica e Vulcanologia, Roma, Italy \\ ${ }^{2}$ Ecole et Observatoire des Science de la Terre, IPG, Strasbourg, France
}

Received: 11 April 2007 - Revised: 6 September 2007 - Accepted: 18 September 2007 - Published: 2 October 2007

\begin{abstract}
After some short test surveys, during the 2004-2005 summer expedition in Antarctica, a geomagnetic French-Italian observatory was installed on the plateau (geographic coordinates: $75.1^{\circ} \mathrm{S}, 123.4^{\circ} \mathrm{E}$; corrected geomagnetic coordinates: $88.9^{\circ} \mathrm{S}, 54.3^{\circ} \mathrm{E}$; UT=LT -8 ) very close to the geomagnetic pole. In this paper we present some peculiarities of the daily variation as observed at this polar cap observatory during the years 2005 and 2006, taking into account the different Loyd seasons and different interplanetary magnetic field conditions. Some interesting results emerge from the analysis, confirming the dependence of the daily variation (and of the associated polar current systems) on the IMF $B_{z}$ and $B_{y}$ components. In particular the analysis showed that different $B_{z}$ conditions correspond to different contribution to daily variation of ionospheric and field aligned currents, while particular $B_{y}$ conditions lead to a time shift of the diurnal variation, indicating an asymmetry with respect to the noon meridian.
\end{abstract}

Keywords. Geomagnetism and paleomagnetism (Time variations, diurnal to secular) - Magnetospheric physics (Polar cap phenomena; Solar wind-magnetosphere interactions)

\section{Introduction}

The ground measurements of magnetic field variations in the polar regions are an important tool for plasma processes analysis (e.g. Lanzerotti et al., 1994; Villante et al., 1997; Yagova et al., 2002, 2004, and the references therein). In particular, the daily variation ( $24 \mathrm{~h}$ period and its harmonics) is generated in the polar cap by two different current systems: $S_{q}^{0}$ (the extrapolation of the mid latitude $S_{q}$ system driven by the ionospheric dynamo) and the polar cap system $S_{q}^{p}$, related to field aligned currents flowing along the geomagnetic

Correspondence to: L. Cafarella

(cafarella@ingv.it) field lines from the magnetosphere to the ionosphere. The $S_{q}^{p}$ system is generated by external sources related to processes in the magnetosphere and its interaction with the Interplanetary Magnetic Field (IMF) (e.g. Ractliffe, 1972; Kivelson and Russel, 1996; Brekke, 1997) and is believed to be the primary source for the high latitude daily variations, especially during local winter when the polar cap ionospheric ionization due to solar radiation is strongly reduced (e.g. Akasofu et al., 1983; Brekke, 1997).

In 1994 France and Italy started a collaboration for opening the permanent scientific station Concordia on the high East Antarctic craton, at Dome C (geographic latitude $75^{\circ} 06^{\prime} \mathrm{S}$, longitude $123^{\circ} 23^{\prime} \mathrm{E}$, about $950 \mathrm{~km}$ away from the coast). Concordia is located $1200 \mathrm{~km}$ away from Mario Zucchelli Station (Italy), $560 \mathrm{~km}$ from Vostok (Russia) and $1100 \mathrm{~km}$ from Dumont D'Urville (France). As a result of this agreement the national Antarctic Programs (IPEV and PNRA respectively) started logistic, technical and scientific activities that initiated with the realization of a summer camp. The permanent Base opened in February 2005, during 2004-2005 summer expedition.

After some preliminary tests, a geomagnetic observatory has been installed at Concordia station at the end of 2004. Variations in the Earth's magnetic field are continuously monitored by a three-axis fluxgate magnetometer along three orthogonal vector components oriented with respect to the local magnetic meridian. The fundamental sampling rate is $1 \mathrm{~s}$ for the vectorial magnetometer and $5 \mathrm{~s}$ for the scalar magnetometer with a data storing rate of $1 \mathrm{~min}$. The total field intensity is measured by an Overhauser magnetometer. Absolute measurements are performed more or less regularly over most of the year. The Antarctic geomagnetic observatory at Concordia Station (DMC) is located near $89^{\circ}$ geomagnetic latitude, deep in the geomagnetic polar cap. The location of the observatory, with a statistical pattern of auroral power flux based on data from the Total Energy Detector (TED) on board the NOAA-15 satellite (courtesy of the U.S.

Published by Copernicus Publications on behalf of the European Geosciences Union. 


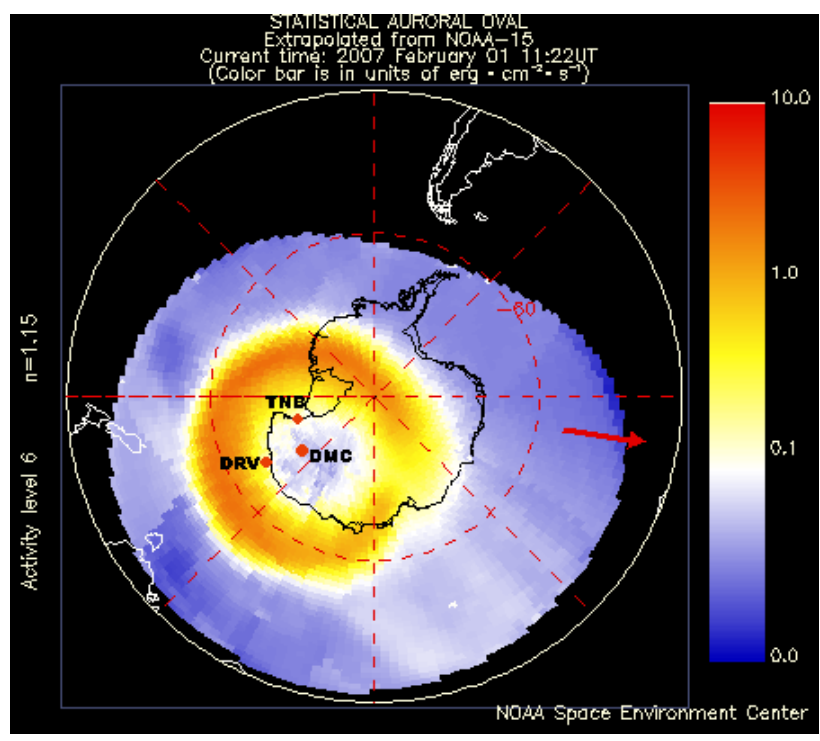

Fig. 1. Current extent and position of the auroral oval in the Southern Hemisphere, extrapolated from measurements taken during a recent polar pass of the NOAA POES satellite (from http: //www.sec.noaa.gov). The position of DMC, TNB and DRV observatories is also reported.

Department of Commerce, NOAA, Space Environment Centre) over Antarctica, are shown in Fig. 1. In the same figure also the position of the Italian and French coastal geomagnetic observatories (indicated as TNB and DRV respectively) are reported.

In a previous paper (Santarelli et al., 2007), we analyzed the daily variation as observed at the geomagnetic observatory TNB (corrected geomagnetic latitude $80.0^{\circ} \mathrm{S}$ ); the analysis, based on fourteen years of data, has shown a pronounced seasonal effect on the amplitude of the diurnal variation, but not on its pattern. A preliminary analysis of the daily variation at DMC, based on a short data set (less than one month) recorded during the 1999-2000 summer campaign was also undertaken; we found that the 24-h variation is well evident on both horizontal elements, with a pronounced amplitude day-to-day variability, related to the global magnetospheric activity level (Lepidi et al., 2003).

In this paper we further investigate the daily variation characteristics as recorded at DMC, i.e. deep in the polar cap. The availability of a longer data set, recorded during the whole 2005 and 2006, allowed to focus our attention on the daily variation seasonal dependence and on the influence of IMF.

In the study of magnetospheric phenomena, especially at high latitude, magnetic local time (MLT) is an important parameter. However, MLT becomes meaningless approaching the geomagnetic pole, where it can not be defined. For this reason, in the following analysis we use the geographic local time (LT) as ordering parameter.

\section{Experimental observations}

The daily variation analysis is performed using hourly average values of the variations of the geomagnetic field horizontal elements $\mathrm{H}$ and $\mathrm{D}$. The total number of used hourly data is about 16000 . The hourly values during the 2005 year, together with the daily averages of the $K_{p}$ index, are shown in Fig. 2 as an example. The presence of a quite regular 24-h variation, whose amplitude strongly varies from day to day, being closely related to $K_{p}$, is clearly evident. During winter, when magnetic activity is lower than summer, the daily variation almost vanishes (see for example the days with $K_{p}=0+$ at the end of May and of June); conversely, during summer the daily variation is always present, although with a smaller amplitude, even when $K_{p}=0$ (at the beginning and the end of December). In order to better investigate this feature, we computed individual power spectra for consecutive 72 -h intervals with a step size of 24-h and obtained, for the whole two-year analyzed period, 557 spectra. These spectra, as expected, show a persistent peak in correspondence of 24-h. As shown in Fig. 3, the logarithmic total horizontal power $(\mathrm{H}$ power $+\mathrm{D}$ power) of the 24 -h peak is well related with the 3day averages of the $K_{p}$ index and the correlation coefficient is slightly higher during winter (similar results are obtained considering the two components separately). Moreover, the slope of the best linear fit considerably increases from summer to winter: in this sense, the plots clearly show that the 24-h power level for the highest $K_{p}$ values (around 4 and more) is comparable in the different seasons, while for very low $K_{p}(<1)$ during winter it is of an order of magnitude lower with respect to summer. Our findings shown in Figs. 2 and 3 on the relation between the diurnal variation amplitude and $K_{p}$ and its seasonal dependence can be attributed to the strong reduction of $S_{q}^{p}$ in the whole year during very quiet magnetospheric conditions and, only in winter, to the absence of the polar extension of the $S_{q}^{0}$, whose amplitude is not related to $K_{p}$.

In Fig. 4 we show the daily distribution of the average hourly values of the $\mathrm{H}$ and $\mathrm{D}$ elements and their standard deviation; the average value of the whole data time series has been subtracted to the data. The analysis has been performed separately for three seasons selected according to the Lloyd criteria (Lloyd, 1861): summer (November, December, January and February), left panels; equinoxes (March, April, September and October), center panels; winter (May, June, July and August), right panels. The plots were obtained by laying one day data on top of another; in this way a single point represents the variation at a fixed LT hour averaged over the whole season; each of these points has been obtained averaging about 200 data.

From Fig. 4 is clear that, independently on the season, the $\mathrm{H}$ variation is symmetric around local noon with a maximum between 11:00 and 12:00 LT, while the D variation is antisymmetric, with a maximum in the local morning and a minimum in the local afternoon (around 07:00 LT and 16:00 LT, 

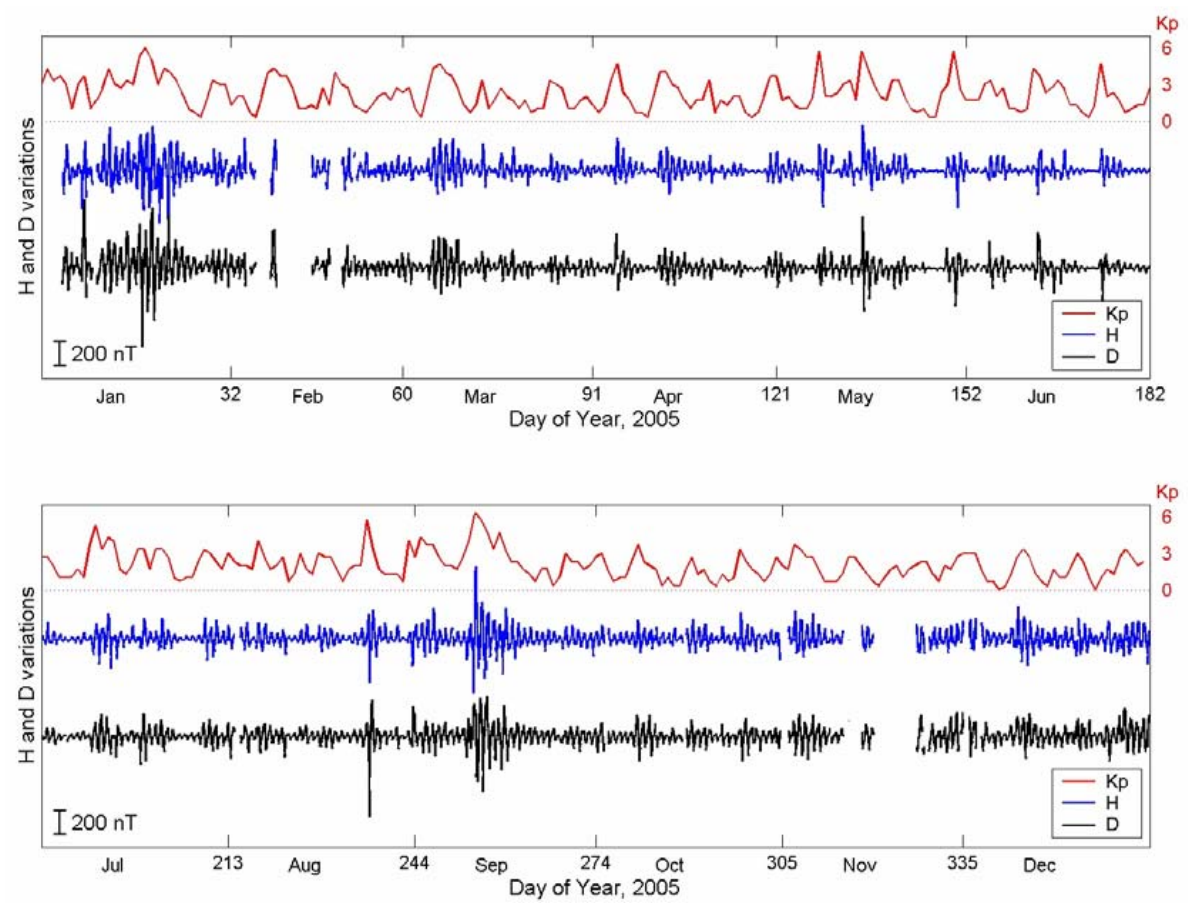

Fig. 2. Hourly values of the variations of the $\mathrm{H}$ and $\mathrm{D}$ components during 2005 , with the daily averages of the $K_{p}$ index.
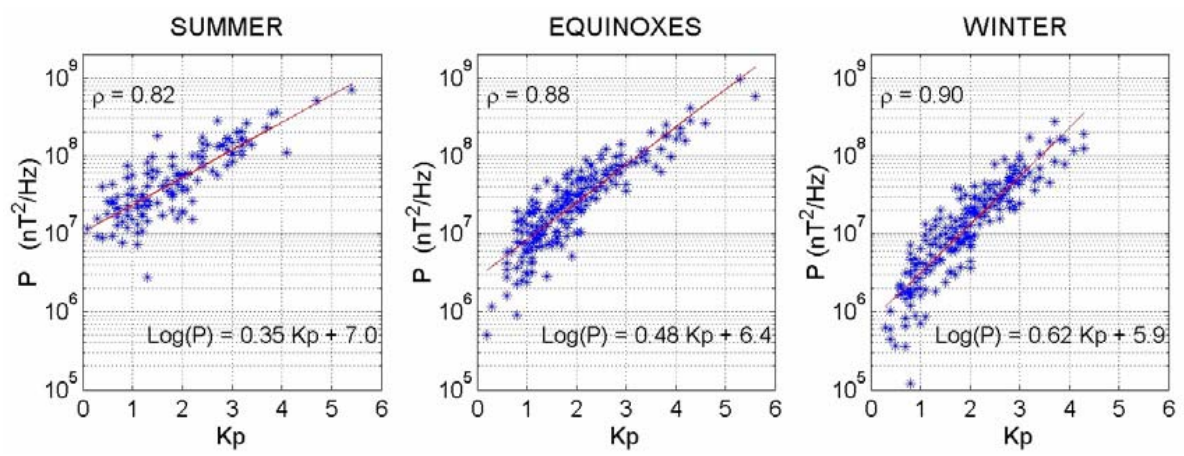

Fig. 3. The total (H+D) 24-h power versus the $K_{p}$ index. The equation of the best linear fit and the value of the correlation coefficient are indicated.

respectively). The pattern of the diurnal variation does not show a seasonal dependence. Conversely, the amplitude of the variation depends on season, being larger during local summer (around $140 \mathrm{nT}$ for both components) and smaller during winter (around $80 \mathrm{nT}$ ). Also the amplitude of the error bars is larger during local summer, indicating a larger day-to-day variability.

In order to investigate solar wind-magnetosphere interaction processes, we used IMF data from ACE spacecraft, in particular the IMF hourly key-parameters from CDAWEB, considering a time delay of $1 \mathrm{~h}$ from spacecraft position (at about $235 R_{E}$ in the Earth-Sun direction) to Earth.

We computed the daily variation pattern for the three seasons separately for positive and negative values of the
IMF $B_{z}$ axial dipole parallel component that correspond to reduced and expanded polar cap conditions, respectively (Iijima, 2000). The results are shown in Fig. 5, in which each point has been obtained averaging about 100 data. It is evident that the amplitude of the variation is definitively larger for $B_{z}<0$, than for $B_{z}>0$; also standard deviation takes on larger values in the condition of $B_{z}<0$. It can also be seen that the amplitude reduction from summer to winter is larger for $B_{z}>0$ (being about a factor 2.5 for both components) than for $B_{z}<0$ (about a factor 1.6). This feature can be interpreted taking into account that for southward IMF conditions the daily variation is mainly due to field aligned currents, which show a moderate reduction in winter; conversely, for northward IMF conditions also the contribution of ionospheric 



Fig. 4. Daily distribution of the average hourly values and their standard deviation for the $\mathrm{H}$ and $\mathrm{D}$ components, considering separately the three Lloyd seasons.

currents, which strongly depend on photo-ionization from the Sun (Friis-Christensen and Wilhjelm, 1975), is important. From Fig. 5 it can also be noted that for $B_{z}<0$ there is also a slight temporal shift of the diurnal variation to earlier hours from summer to winter.

In Fig. 6 we show the diurnal variation for $B_{z}>0$ and $\left|B_{y}\right|>B_{z}$ (where $B_{y}$ is the east-west IMF component), separately for positive and negative $B_{y}$ (corresponding respectively to $B_{y}>B_{z}$ and $B_{y}<-B_{z}$, respectively); each point has been obtained averaging about $100-150$ data. It is interesting to point out that for negative $B_{y}$ the pattern of the diurnal variation is shifted earlier of about $3 \mathrm{~h}$ with respect to positive $B_{y}$. For example, the $\mathrm{H}$ component maximum is at 09:0010:00 LT for $B_{y}<0$ and at 12:00-13:00 LT for $B_{y}>0$, and the D component maximum is at 05:00 LT and 09:00 LT, respectively. It is also evident that for both elements the amplitude and standard deviation of the diurnal variation is larger, almost by a factor two, for negative $B_{y}$.

This result confirms that the polar cap electric current system $S_{q}^{p}$ (generated by external sources related to processes in the magnetosphere and its interaction with IMF) deeply depends on $B_{y}$. In order to better investigate this phenomenon, we analyzed also the seasonal dependence of the daily variation for positive $\mathrm{B}_{z}$ and negative $B_{y}$ conditions (Fig. 7). In this case the curves are not very regular, due to the poorer statistics (each point has been obtained averaging about 3040 data); however the results indicate that the shift toward earlier hours of the diurnal variation for $B_{y}<0$ is more pro- nounced during winter, when the solar irradiation is completely absent and the electric current polar system is poorly influenced by the ionospheric $S_{q}$ electric current system.

\section{Summary}

The diurnal variation of geomagnetic field elements is a well known phenomenon that has been extensively studied especially at mid latitudes, where $S_{q}$ electric current system is driven by the ionospheric dynamo and consists of two current vortices, centered at about $40^{\circ}$ latitude $\mathrm{N}$ and $\mathrm{S}$, respectively. An equivalent electric current system is used in polar region to represent the source of the observed field. This current system is called $S_{q}^{p}$ (Solar quiet polar) and is related to magnetospheric convection generating a quasi permanent current system steadily oriented respect to the Sun-Earth line. In this paper data from Dome C, a polar cap observatory, were analysed for the years 2005 and 2006 in order to investigate daily variation dependence on magnetospheric and IMF conditions.

The first result found is the evidence of a strong relation between the daily variation amplitude and the $K_{p}$ index during the whole year. This result confirms our previous findings obtained from a short data set during summer (Lepidi et al., 2003). Moreover, we found that the relation between the diurnal variation amplitude and $K_{p}$ shows a seasonal dependence, in that the correlation coefficient is higher during winter and the slope of the best linear fit considerably increases 

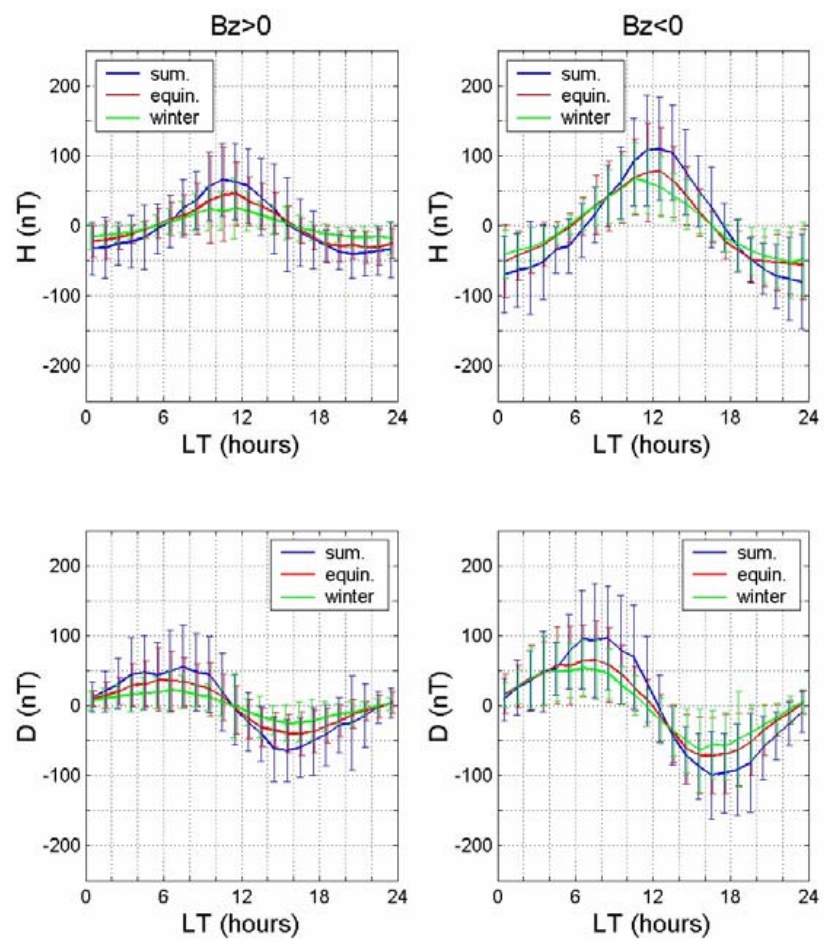

Fig. 5. Daily distribution of the average hourly values and their standard deviation for the $\mathrm{H}$ and $\mathrm{D}$ components, separately for the three Lloyd seasons, for northward (left panels) and southward (right panels) IMF conditions.

from summer to winter: indeed, for high $K_{p}$ conditions, the amplitude of the daily variation is comparable in the different seasons; conversely when the $K_{p}$ index attains minimum values, the daily variation is much more reduced during winter, being of an order of magnitude smaller than during summer. These findings indicate that in the polar region the amplitude of the daily variation is strongly dependent on high latitude electric current system, while the contribution of the polar extension of $S_{q}$, whose amplitude is not related to $K_{p}$, is negligible, especially during winter.

It was also found that the amplitude of the daily variation, which decreases from summer to winter, strongly depends on IMF conditions, being larger when $B_{z}<0$ (expanded polar cap region; Iijima, 2000). Moreover the amplitude reduction from summer to winter is larger for $B_{z}>0$, indicating that in this case the contribution of ionospheric currents (strongly dependent on photo-ionization from the Sun) could be important, while for $B_{z}<0$ the key role is played by field aligned currents, with a moderate seasonal variation.

At high latitude also the east-west IMF component, $B_{y}$, plays a main role in determining geomagnetic variations. Indeed, for $B_{z}<0$, the $B_{y}$ component creates an asymmetry about the noon-midnight meridian in the evolution of newly opened field lines (Svalgaard-Mansurov effect; see for example Zhou et al., 2000; Stubbs et al., 2001). Moreover,
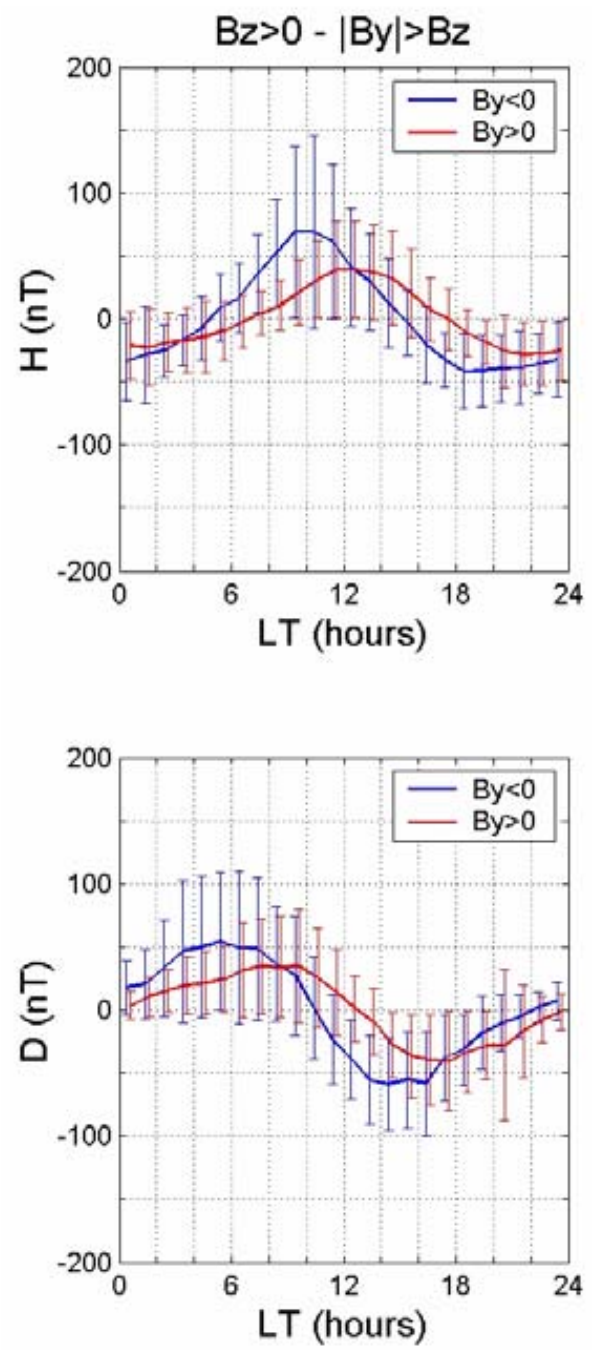

Fig. 6. Daily distribution of the average hourly values and their standard deviation for the $\mathrm{H}$ and $\mathrm{D}$ components, for $B_{z}>0$ and $\left|B_{y}\right|>B_{z}$, separately for positive and negative $B_{y}$.

more recently many authors have shown the importance of $B_{y}$ also for positive $B_{z}$ conditions: in this case, the existence and the geometry of the polar cap, as well as the local current geometry, is deeply connected to the value of the $B_{y}$ component (Vennstrom et al., 2002, 2005; Park et al., 2006). Indeed, the introduction of an eastward IMF component, during northward IMF conditions, gradually opens the magnetosphere poleward of the cusp (as originally suggested by Burch, 1973), changing the pattern of the ionospheric currents that will be located asymmetrically respect to the noon-midnight meridian; in particular, in the Southern Hemisphere, dusk (dawn) side currents are enhanced for positive (negative) $B_{y}$ and this asymmetry increases when $B_{y}$ increases with respect to $B_{z}$ (Vennstrom et al., 2005). In this sense our results have shown that, for positive $B_{z}$, when $B_{y}<0$ the diurnal variation is shifted earlier of about $3 \mathrm{~h}$ with 

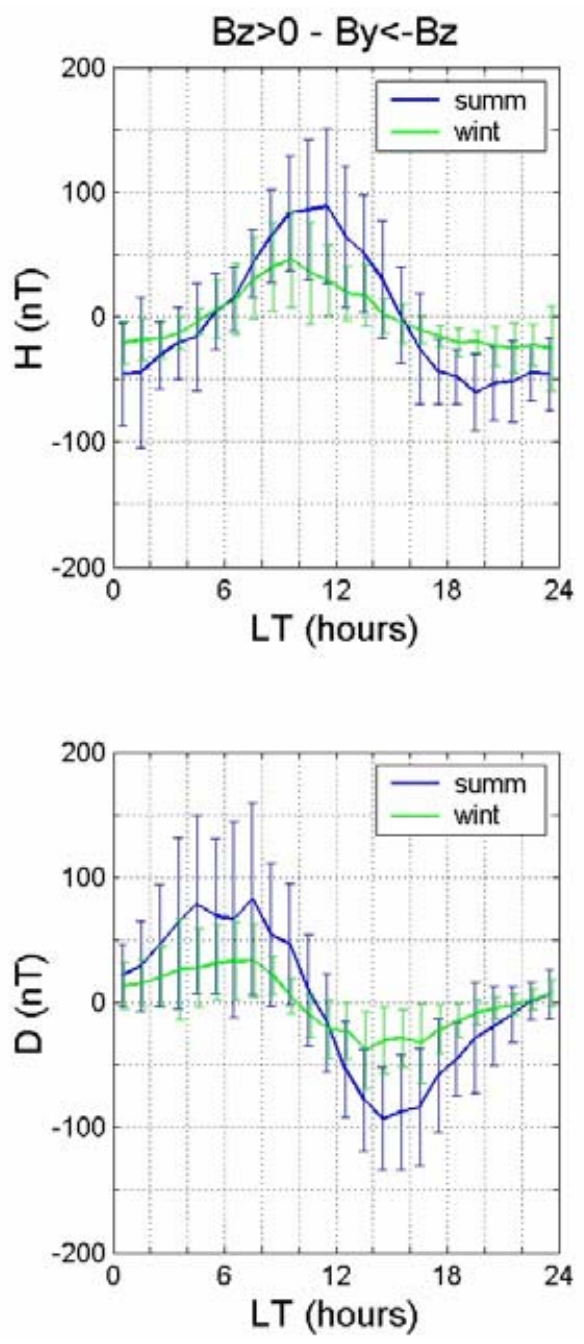

Fig. 7. Daily distribution of the average hourly values and their standard deviation for the $\mathrm{H}$ and $\mathrm{D}$ components, for $B_{z}>0$ and $B_{y}<-B_{z}$, separately for summer and winter seasons.

respect to $B_{y}>0$ and this time shift is even more evident during winter. Moreover the amplitude of the diurnal variation is larger, almost by a factor two, for $B_{y}<0$ when in general the magnetic activity in the high latitude Southern Hemisphere tends to be higher (Nishida, 1978).

Acknowledgements. The research activity at DMC is supported by Italian PNRA and French IPEV. The work of M. Pietrolungo and L. Santarelli was supported by a PNRA fellowship.

The authors would like to thank the two referees for their helpful comments and suggestions on the manuscript.

Topical Editor I. A. Daglis thanks K. Yumoto and another anonymous referee for their help in evaluating this paper.

\section{References}

Akasofu, S. I., Ahn, B. H., and Romick, G. J.: A study of the polar current system using the IMS meridian chains of magnetometers, Space Sci. Rev., 36, 337-413, 1983.

Brekke, A.: Physics of the upper polar atmosphere, John Wiley \& Sons Ltd in association with Praxis Publishing Ltd, p. 491, 1997.

Burch, J. L.: Rate of erosion of dayside magnetic flux based on a quantitative study of polar cusp latitude on the interplanetary magnetic field, Eos T. Am. Geophys. Un., 54, 1286-1286, 1973.

Friis-Christensen, E. and Wilhjelm, J.: Polar cap currents for different directions of the interplanetary magnetic field in the $\mathrm{Y}-\mathrm{Z}$ plane, J. Geophys. Res., 80, 1248-1260, 1975.

Kivelson, M. G. and Russell, C. T.: Introduction to Space Physics, Cambridge University Press, p. 568, 1996.

Iijima, T.: Field-aligned currents in geospace: substance and significance, Magnetospheric Current Systems, edited by: Ohtani, S.-I., Fujii, R., Hesse, M., Lysak, R. L., Gephysical Monograph, 118, 107-129, 2000.

Lanzerotti, L. J., Meclennan, L. V., and Medford, L. V.: Inferred quasi-steady ionospheric neutral winds and electrical currents at $79^{\circ}$ south latitude in austral summer condition, Geophys. Res. Lett., 21, 217-220, 1994.

Lepidi, S., Cafarella, L., Francia, P., Meloni, A., Palangio, P., and Schott, J. J.: Low frequency geomagnetic field variations at Dome C (Antartica), Ann. Geophys., 21, 923-932, 2003, http://www.ann-geophys.net/21/923/2003/.

Lloyd, H.: On earth-currents, and their connexion with the diurnal changes of the horizontal magnetic needle, Trans. Roy. Irish Acad., 24, 115-141, 1861.

Nishida, A.: Geomagnetic diagnosis of the magnetosphere, Springer-Verlag, 1978.

Park, K. S., Ogino, T., and Walker, R. J.: On the importance of antiparallel reconnection when the dipole tilt and IMF $B_{y}$ are nonzero, J. Geophys. Res., 111, A05202, doi:10.1029/2004JA010972, 2006.

Ratcliffe, J. A.: An introduction to the ionosphere and magnetosphere, Com. Uni Press, Cambridge, p. 256, 1972.

Santarelli, L., Cafarella, L., Lepidi, S., Di Mauro, D., Meloni, A., and Palangio, P.: Fourteen years of geomagnetic daily variation at Mario Zucchelli Station (Antarctica), Ann. Geophys.-Italy, 50, 227-234, 2007.

Stubbs, T. J., Lockwood, M., Cargill, P., Fennell, J., Grande, M., Kellett, B., Perry, C., and Rees, A.: Dawn-dusk asymmetry in particles of solar wind origin within the magnetosphere, Ann. Geophys., 19, 1-9, 2001,

http://www.ann-geophys.net/19/1/2001/.

Vennerstrom, S., Moretto, T., Olsen, N., Friis-Christensen, E., and Stampe, A. M.: Field aligned currents in the dayside cusp and polar cap region during northward IMF, J. Geophys. Res., 107, A009162, doi:10.1029/2001JA009162, 2002.

Vennerstrom, S., Moretto, T., Rastatter, L., and Raeder, J.: Field-aligned currents during northward interplanetary magnetic field: Morphology and causes, J. Geophys. Res., 110, A06205, doi:10.1029/2004JA010802, 2005.

Villante, U., Lepidi, S., Francia, P., Meloni A., and Palangio, P.: Long period geomagnetic field fluctuations at Terra Nova Bay (Antarctica), Geophys. Res. Lett., 24, 1443-1446, 1997.

Yagova, N. V., Lanzerotti, L. J., Villante, U., Pilipenko, V. A., Lepidi, S., Francia, P., Papitashvili, V. O., and Rodger, A. S.: ULF 
Pc5-6 magnetic activity in the polar cap as observed along a geomagnetic meridian in Antarctica, J. Geophys. Res., 107, SMP22, doi:10.1029/2001JA900143, 2002.

Yagova, N. V., Pilipenko, V. A., Lanzerotti, L. J., Engebretson, M. J., Rodger, A. S., Lepidi S., and Papitashvili, V. O.: Two-dimensional structure of long-period pulsations at polar latitudes in Antarctica, J. Geophys. Res., 109, A03222, doi:10.1029/2003JA010166, 2004.
Zhou, X. W., Russell, C. T., Le Fuselier, S. A., and Scudder, J. D.: Solar wind control of the polar cusp at high altitude, J. Geophys. Res., 105, 245-251, doi:10.1029/1999JA900412, 2000. 